\title{
CHEMICAL POTENTIAL AS A DETECTOR OF PHASE TRANSITIONS IN SOLIDS
}

\author{
M. $\operatorname{Matlak}^{a *}$, E. Goslawska ${ }^{a}$, B. Grabiec $^{a}$ AND Kh. Eid ${ }^{b}$ \\ ${ }^{a}$ Institute of Physics, Silesian University, Uniwersytecka 4, 40-007 Katowice, Poland \\ ${ }^{b}$ Physics Department, Faculty of Education, Ain-Shams University, Roxy, Cairo, Egypt
}

We show that the chemical potential exhibits small but distinct kinks at all critical temperatures as the evidence for phase transitions in the electronic system, structural phase transitions included. In the case of, at least, two kinds of interacting electrons average occupation numbers exhibit the same behavior.

PACS numbers: $74.70 .-\mathrm{b}, 75.30 . \mathrm{Kz}, 75.40 . \mathrm{Cx}$

We consider phase transitions from ferromagnet to paramagnet $(\mathrm{F}-\mathrm{P})$, from superconductor to normal system $(\mathrm{S}-\mathrm{N})$ and we investigate phase transitions of reentrant type $(R)$ using a generalization of the model investigated in Ref. [1].

The Hamiltonian of the model (three hybridized bands) can be written in the following form (grand canonical ensemble):

$$
\begin{aligned}
\bar{H}= & -\frac{1}{2} \sum_{i \neq j} J_{i, j} \boldsymbol{S}_{i} \cdot \boldsymbol{S}_{j}+\sum_{\alpha} t_{0}^{(\alpha)} \sum_{i, \sigma} n_{i, \sigma}^{(\alpha)}+\sum_{\alpha} \sum_{i \neq j, \sigma} t_{i, j}^{(\alpha)} c_{i, \sigma}^{+(\alpha)} c_{j, \sigma}^{(\alpha)} \\
& +\sum_{\alpha<\beta} V^{(\alpha, \beta)} \sum_{i \neq j, \sigma}\left(c_{i, \sigma}^{+(\alpha)} c_{j, \sigma}^{(\beta)}+c_{j, \sigma}^{+(\beta)} c_{i, \sigma}^{(\alpha)}\right) \\
& -\sum_{\alpha} \frac{g^{(\alpha)}}{2} \sum_{i}\left[S_{i}^{+} c_{i, \downarrow}^{+(\alpha)} c_{i, \uparrow}^{(\alpha)}+S_{i}^{-} c_{i, \uparrow}^{+(\alpha)} c_{i, \downarrow}^{(\alpha)}+S_{i}^{z}\left(n_{i, \uparrow}^{(\alpha)}-n_{i, \downarrow}^{(\alpha)}\right)\right] \\
& +\sum_{\alpha} \sum_{i \neq j} R_{i, j}^{(\alpha)} c_{i, \uparrow}^{+(\alpha)} c_{j, \downarrow}^{+(\alpha)} c_{j, \downarrow}^{(\alpha)} c_{i, \uparrow}^{(\alpha)}-\mu \sum_{\alpha} \sum_{i, \sigma} n_{i, \sigma}^{(\alpha)},
\end{aligned}
$$

the index $\alpha$ numbers the bands $(\alpha=1,2,3)$ and $\mu$ is the chemical potential. The Hamiltonian (1) describes the magnetic interaction (localized $4 f$-spins), three hybridized bands and magnetic interactions between band electrons and localized $4 f$-spins, as well as, the intersite Cooper pairs interaction. (cf. e.g. [2]) between' conduction electrons. To calculate the chemical potential $\mu$ we use the constraint $\sum_{\alpha} n^{(\alpha)}=\bar{n}\left(n^{(\alpha)}=\sum_{\sigma}\left\langle n_{\sigma}^{(\alpha)}\right\rangle\right)$, where $\bar{n}$ is the average number of electrons per

\footnotetext{
*e-mail:matlak@server.phys.us.edu.pl
} 
magnetic Re-ion. Assuming the spin length $s=1 / 2$ and performing the calculations similarly to Ref. [1] (MFA ${ }^{\dagger}$, Green's function method [3]) and using the exact formula for the density of states (sc lattice, see [4]), we can study the following phase transitions: (i) F-P $\left(R_{i, j}^{(\alpha)}=0\right)$, (ii) S-N $\left(J_{0}=g^{(\alpha)}=0\right)$, (iii) R transitions with three critical temperatures $T_{s_{1}}, T_{\mathrm{c}}$, and $T_{s_{2}}\left(R_{i, j}^{(\alpha)} \neq 0, J_{0} \neq 0, g^{(\alpha)} \neq 0\right)$. In all cases we have introduced a possibility of a structural phase transition (see e.g. $[5,6]$ ) at $T=T_{0}$ assuming a small change of the bandwidth $W$ at $T=T_{0}\left(W_{T \leq T_{0}}=W+\delta, W_{T>T_{0}}=W\right.$, where $\delta$ is very small $)$.

In Fig. 1a-c we present the results $\left(T_{0}=6 \mathrm{~K}\right)$ for $n=3$. The results for $n=2$ (one empty band, $t^{(3)}=100 \mathrm{eV}$ ) are qualitatively very similar to the case $n=3$. We present a very interesting case $n=1 \mathrm{in} \mathrm{Fig.} 2 \mathrm{a}-\mathrm{c}\left(T_{0}=10 \mathrm{~K}\right)$. We see that all critical temperatures of the system can be well localized from
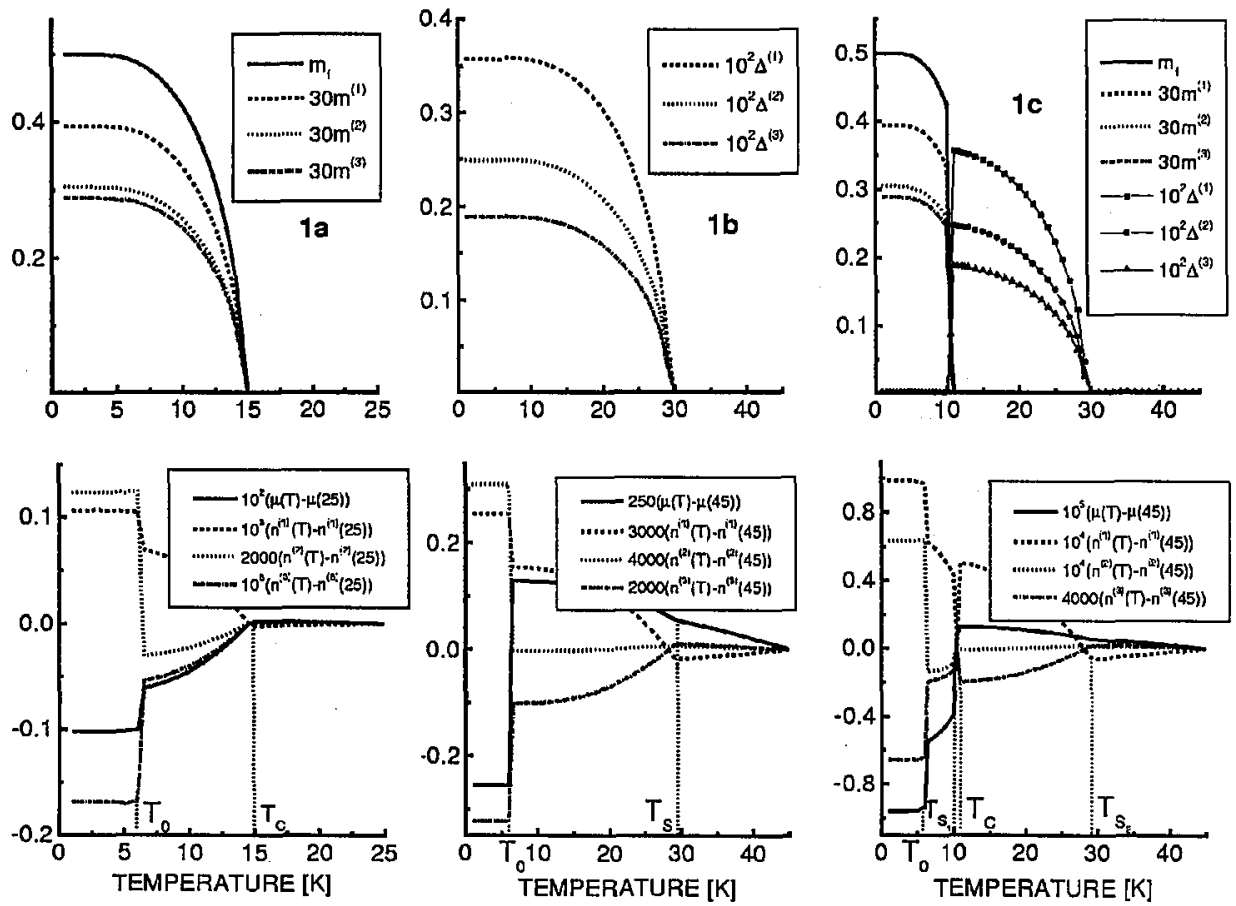

Fig. 1. Phase transitions for $n=3$; upper plots: order parameters (magnetizations $m_{f}$ and $m^{(\alpha)}$ and superconducting order parameters $\Delta^{(\alpha)}$ ) vs. temperature; lower plots: chemical potential $\mu[\mathrm{eV}]$ and average occupation numbers $n^{(\alpha)}$ against temperature. Model parameters (Fig. 1c): $\bar{n}=0.6, J_{0}=\sum_{j} J_{i, j}=0.0001 \mathrm{eV}, g^{(1)}=g^{(2)}=g^{(3)}=$ $0.075 \mathrm{eV}, t_{0}^{(1)}=0, t_{0}^{(2)}=0.075 \mathrm{eV}, t_{0}^{(3)}=0.1 \mathrm{eV}, W^{(1)}=W=0.55 \mathrm{eV}, \delta=0.005 \mathrm{eV}$, $\delta_{2}=W^{(2)} / W=0.9, \delta_{3}=W^{(3)} / W=0.8, V^{(1,2)}=0.07 \mathrm{eV}, V^{(1,3)}=0.08 \mathrm{eV}, V^{(2,3)}=$ $0.075 \mathrm{eV}, R_{0}^{(1)}=0.22 \mathrm{eV}, R_{0}^{(2)}=0.19 \mathrm{eV}, R_{0}^{(3)}=0.16 \mathrm{eV}\left(R_{0}^{(\alpha)}=R_{i, j}^{(\alpha)} ; i, j-\right.$ n.n. $)$. In (a) we put $R_{0}^{(\alpha)}=0$ and in (b) we put $J_{0}=g^{(\alpha)}=0$.

${ }^{\dagger}$ We consider, for simplicity, the single-domain case with homogeneous magnetization. 

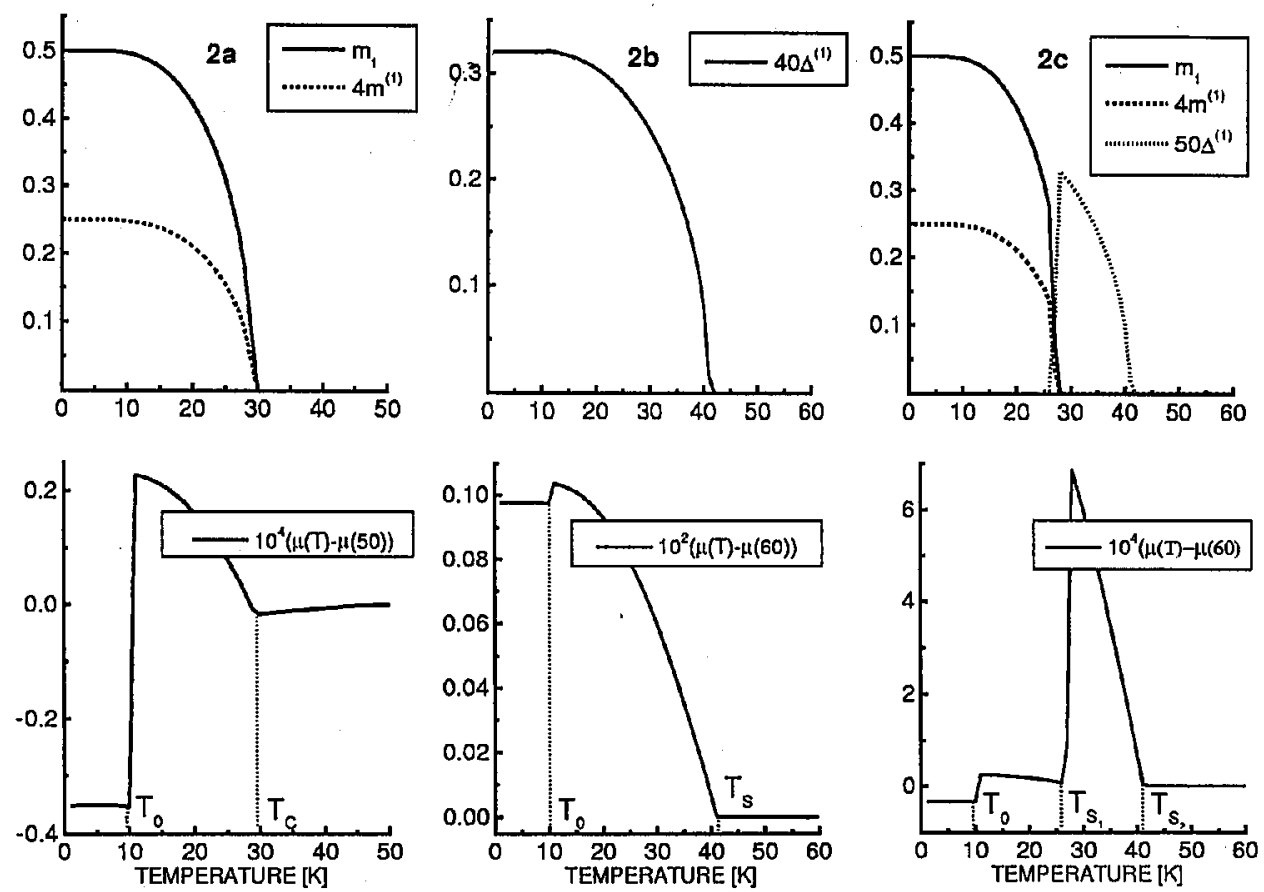

Fig. 2. The same as in Fig. 1 but for $n=1$ when $t_{0}^{(2)}=200 \mathrm{eV}, t_{0}^{(3)}=100 \mathrm{eV}$ (two empty bands). The other parameters are the same as in Fig. 1a,b,c.
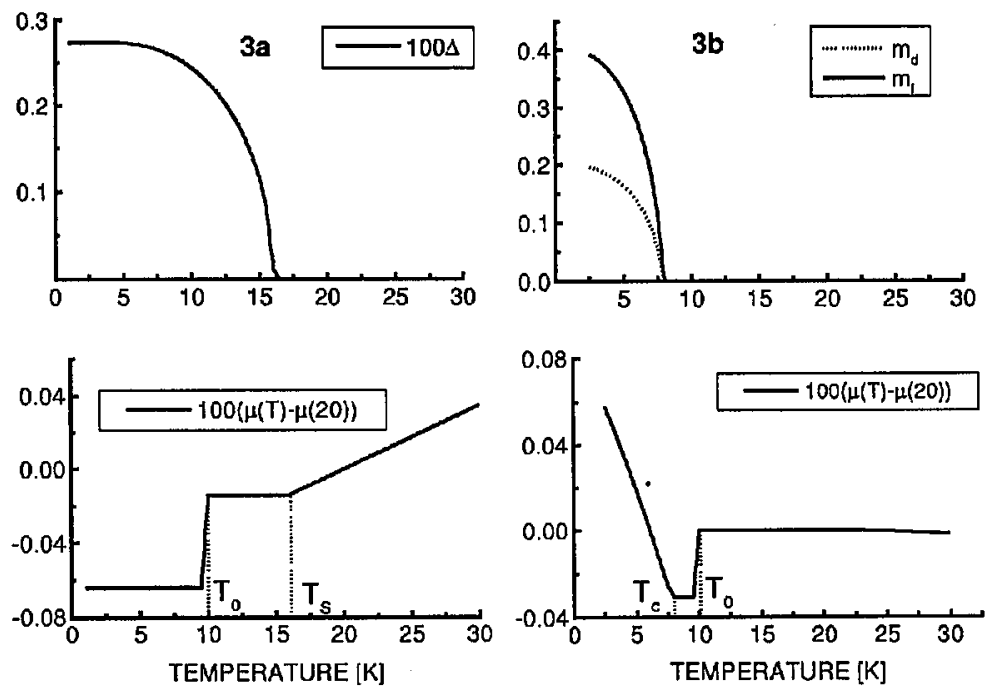

Fig. 3. The exact solutions of the model (2) (a) and (3) (b). Common model parameters: $\bar{n}=0.6, t_{T \leq T_{0}}=0.0825 \mathrm{eV}, t_{T>T_{0}}=0.082 \mathrm{eV}$. (a) $J_{0}=0.003 \mathrm{eV}, g=0.3 \mathrm{eV}$ and (b) $R=0.011 \mathrm{eV}$. 
the kinks in the temperature dependence of the chemical potential. The effect of the critical electron redistribution [7], present for $n=2,3$ cannot take place for $n=1\left(n^{(1)}=\bar{n}=\right.$ const). Let us also note that the lower plot in Fig. $2 \mathrm{~b}$ is very similar to the experimentally measured temperature dependence of the chemical potential for $\mathrm{YBa}_{2} \mathrm{Cu}_{3} \mathrm{O}_{7-\delta}$ high- $T_{\mathrm{c}}$ superconductor (see Fig. 2 in Ref. [8]).

Let us also consider two exactly solvable models (grand canonical ensemble)

$$
\begin{gathered}
\bar{H}=-a \sum_{i} S_{i}^{z}-\frac{g}{2} \sum_{i}\left[S_{i}^{+} c_{i, \downarrow}^{+} c_{i, \uparrow}+S_{i}^{-} c_{i, \uparrow}^{+} c_{i, \downarrow}+S_{i}^{z}\left(n_{i, \uparrow}-n_{i, \downarrow}\right)\right] \\
-t \sum_{i \neq j, \sigma} c_{i, \sigma}^{+} c_{j, \sigma}-\mu \sum_{i, \sigma} n_{i, \sigma}, \quad\left(i, j=1,2 ; a=m_{f} J_{0}\right), \\
\bar{H}=-t \sum_{i \neq j, \sigma} c_{i, \sigma}^{+} c_{j, \sigma}-R \Delta \sum_{i \neq j}\left(c_{j, \downarrow} c_{i, \uparrow}+c_{i, \uparrow}^{+} c_{j, \downarrow}^{+}\right)-\mu \sum_{i, \sigma} n_{i, \sigma}, \\
\left(i, j=1,2 ; \Delta=\left\langle c_{1, \downarrow} c_{2, \uparrow}\right\rangle=\left\langle c_{2, \downarrow} c_{1, \uparrow}\right\rangle\right) .
\end{gathered}
$$

To calculate $\mu$ we use the constraint $\left\langle n_{i, \uparrow}\right\rangle+\left\langle n_{i, \downarrow}\right\rangle=\bar{n}(i=1,2)$. The results $\left(T_{0}=10 \mathrm{~K}\right.$ ) are depicted in Fig. 3a (model (2)) and Fig. 3b (model (3)). Also in the case of exactly solvable models the critical temperatures can easily be identified from the kinks of the chemical potential which clearly suggest a possibility to use the chemical potential as a detector in a broad context of phase transitions in réal solids.

\section{References}

[1] E. Gosławska, M. Matlak, Phys. Status Solidi B 207, 469 (1998).

[2] R. Micnas, J. Ranninger, S. Robaszkiewicz, Rev. Med. Phys. 62, 113 (1990).

[3] A.A. Abrikosov, L.P. Gorkov, I.E. Dzyaloshinskii, Quantum Field Theoretical Methods in Statistical Physics, Pergamon Press, Oxford 1965.

[4] R. Jelitto, J. Phys. Chem. Solids 30, 609 (1969).

[5] A.D. Bruce, R.A. Cowley, Structural Phase Transitions, Taylor \& Francis, London 1981.

[6] P.M. Woodward, Acta Crystallogr. B 53, 32, 44 (1997).

[7] M. Matlak, M. Pietruszka, Physica C 311, 151 (1999).

[8] G. Rietveld, N.Y. Chen, D. van der Marel, Phys. Rev. Lett. 69, 2578 (1992). 